\title{
Sobre los cuasi-nodos en las oscilaciones de cuerdas reales
}

\author{
(About the quasi-nodes in the real strings's oscillations)
}

\author{
Danilo Gómez y Reinaldo Welti ${ }^{1}$ \\ ${ }^{1}$ Facultad de Ciencias Exactas e Ingeniería, Universidad Nacional de Rosario \\ Recebido em 05/01/04; Aceito em 16/03/04
}

\begin{abstract}
En este trabajo se estudian los efectos que provoca una fuerza viscosa sobre las oscilaciones forzadas de una cuerda tensa impulsada periódicamente en uno de sus extremos.

Palabras-clave: ondas estacionarias, cuerdas disipativas, resonancia, nodos, transferencia de energía.
\end{abstract}

In this paper we study the effects of a viscous force on the oscillations of a tense rope that it is impelled periodically in one of their ends.

Keywords: standing waves, dissipative string, resonance, nodes, energy transfer.

\section{Introducción}

Cuando se realiza en el laboratorio la experiencia de las oscilaciones forzadas de una cuerda se advierte que en los "nodos" la amplitud no es nula. Si los estudiantes que trabajan con esta experiencia son buenos observadores comentan también que la amplitud de oscilación es máxima en el nodo más próximo al extremo forzado y a partir de ahí disminuyen linealmente hasta llegar al extremo fijo (ver Figura 1 y 2).

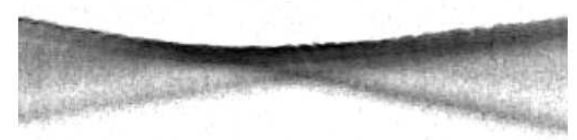

Figura 1: Cuasi-nodo próximo al vibrador.

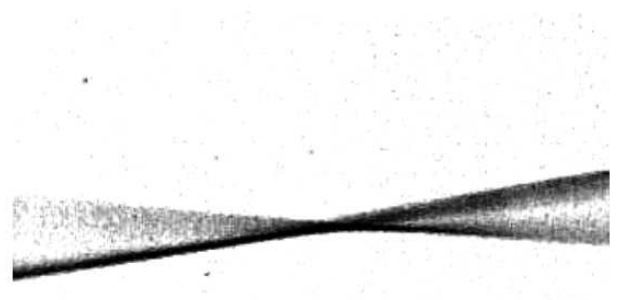

Figura 2: Cuasi-nodo próximo al extremo fijo.
Esta interesante observación permite analizar con ellos sobre los efectos de la disipación de energía sobre el patrón de oscilación de la cuerda. El instructor generalmente lo explica diciendo que para mantener estacionaria las oscilaciones a lo largo de toda la cuerda la energía que entrega el vibrador debe viajar a lo largo de la misma para compensar las pérdidas que se producen y como esta energía debe pasar a través de los "nodos", éstos no pueden tener una amplitud nula.

Sin embargo, solo algunos textos que tratan las oscilaciones en cuerdas emplean este razonamiento cualitativo y ninguno desarrolla una teoría que tenga en cuenta la disipación. El objetivo de este trabajo es presentar una teoría, relativamente simple, de las vibraciones forzadas de una cuerda con pérdidas, que puede ser muy útil para los profesores de física básica que presentan este tema en el aula o en el laboratorio. Esta teoría explica la amplitud de oscilación no nula de los cuasi-nodos y la disminución de su amplitud cuando más cerca están del extremo fijo. Se muestra también que los picos de la curva de amplitud de respuesta, en un entorno de la frecuencia de resonancia, son lorentzianas. Este comportamiento de la amplitud de respuesta ha sido comprobado por nuestras mediciones. La medición del ancho de banda en frecuencia de estas lorentzianas permite medir la constante de atenuación de la onda y su eventual dependencia de la frecuencia.

\section{Ecuación de ondas en una cuerda con pérdidas}

La ecuación para los desplazamientos transversales $\Phi$ de una cuerda flexible, sin disipación, que está tendida paralelamente al eje $x$, es la ecuación de ondas clásica:

\footnotetext{
${ }^{1}$ Enviar correspondência para Danilo Gómez y Reinaldo Welti. E-mail: weltreb@amet.com.ar.
}

Copyright by the Sociedade Brasileira de Física. Printed in Brazil. 


$$
c^{2} \frac{\partial^{2} \Phi}{\partial x^{2}}=\frac{\partial^{2} \Phi}{\partial t^{2}}
$$

donde $c=\sqrt{T / \mu}$ es la velocidad de la propagación, $T$ la tensión de la cuerda y $\mu$ su masa por unidad de longitud.

La mayoría de los sistemas reales están afectados por fuerzas de amortiguamiento que hacen que la onda que se propaga a través del medio pierda su energía. Para tener en cuenta las fuerzas disipativas en una cuerda elástica, se agrega a la fuerza de restitución, una fuerza viscosa efectiva[1], por unidad de longitud, igual a $-b \partial \Phi / \partial t$ donde $b$ es una constante. Esta fuerza introduce un nuevo término en la ecuación de ondas, que ahora se escribe de la forma:

$$
c^{2} \frac{\partial^{2} \Phi}{\partial x^{2}}-\Gamma \frac{\partial \Phi}{\partial t}=\frac{\partial^{2} \Phi}{\partial t^{2}}
$$

donde $\Gamma=b / \mu$.

Como la ecuación (1) es una ecuación lineal, podemos obtener soluciones armónicas de la forma

$$
\Phi(x, t)=\widetilde{\Phi}(x) e^{i \omega t}
$$

donde $\widetilde{\Phi}(x)$ es la amplitud compleja que depende de la posición $x$ sobre la cuerda. La ecuación diferencial que debe satisfacer se encuentra al sustituir $\widetilde{\Phi}(x) e^{i \omega t}$ por $\Phi(x, t)$ en (1). Después de cancelar el factor $e^{i \omega t}$, encontramos

$$
c^{2} \frac{\partial^{2} \widetilde{\Phi}(x)}{\partial x^{2}}+\left(\omega^{2}-i \Gamma \omega\right) \widetilde{\Phi}(x)=0
$$

La solución general de la ecuación (3) es

$$
\widetilde{\Phi}(x)=A e^{-\gamma x}+B e^{\gamma x}
$$

donde $A$ y $B$ son dos constantes complejas arbitrarias, mientras que

$$
\gamma=\frac{1}{c} \sqrt{i \Gamma \omega-\omega^{2}}=\alpha+i \beta
$$

es la constante de propagación compleja, $\alpha$ la constante de atenuación y $\beta$ la constante de fase.

Si multiplicamos (4) por $e^{i \omega t}$ obtenemos

$$
\Phi(x, t)=\widetilde{\Phi}(x) e^{i \omega t}=A e^{-\alpha x} e^{i(\omega t-\beta x)}+B e^{i(\omega t+\beta x)}
$$

La ecuación (6) representa a dos ondas, una que se propaga hacia las $x$ positivas y otra hacia las $x$ negativas, respectivamente, con amplitudes que decrecen a medida que estas ondas se van propagando.

Para encontrar $\alpha$ y $\beta$ debemos resolver el sistema de ecuaciones:

$$
\begin{aligned}
\frac{\omega^{2}}{c^{2}} & =\beta^{2}-\alpha^{2} \\
\Gamma \omega & =2 c^{2} \alpha \beta
\end{aligned}
$$

que se deduce de la ecuación (5).

En la ausencia de pérdidas, $\alpha=0$ y $\beta=\omega / c=k$, donde $k$ es el número de onda o la constante de fase en ausencia de pérdidas.

Si las pérdidas son pequeñas $(\Gamma \ll \omega)$, se tiene que

$$
\begin{aligned}
& \beta \approx \omega / c \\
& \alpha \approx \Gamma / 2 c
\end{aligned}
$$

\subsection{Vibración armónica forzada de la cuerda}

En nuestra experiencia, la cuerda de longitud $L$ está fija en su extremo $x=0$, mientras que su extremo $x=L$ está conectado a un dispositivo (un vibrador) que oscila transversalmente con una amplitud constante $\Phi_{0}$ y con una frecuencia $\omega$ que puede variar libremente. El dispositivo experimental que se utilizó se muestra esquemáticamente en la Fig. 3.

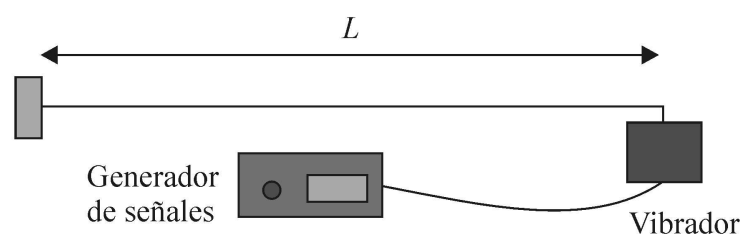

Figura 3: Arreglo experimental para la excitación de los modos de vibración de una cuerda tensa.

El desplazamiento transversal de la cuerda $\Phi(x, t)$ que viene dado por la ecuación (6), es la solución de la ecuación de ondas (1), en el intervalo $0<x<L$. La amplitud compleja $\widetilde{\Phi}(x)$ de esta solución debe satisfacer las siguientes condiciones de borde:

$$
\widetilde{\Phi}(0)=0
$$

$$
\widetilde{\Phi}(L)=\Phi_{0}
$$

La condición (9) impone que $A=-B$, de modo que la (4) puede escribirse de la forma:

$$
\widetilde{\Phi}(x)=B\left(e^{\gamma x}-e^{-\gamma x}\right)=2 B \sinh (\gamma x)
$$

donde sinh es la función seno hiperbólico.

De la condición de borde (10) se deduce que

$$
B=\frac{\Phi_{0}}{2 \sinh (\gamma L)}
$$

Reemplazando (12) en (11) obtenemos:

$$
\widetilde{\Phi}(x)=\Phi_{0} \frac{\sinh (\gamma x)}{\sinh (\gamma L)}
$$

En la ausencia de pérdidas, $\alpha=0$ y $\gamma=i k$, donde $k=$ $\omega / c$ es la constante de fase de un medio sin pérdidas. Como $\sinh (i \omega x / c)=i \sin (\omega x / c)$ la ecuación (13) se reduce a la expresión que deduce French[2].

$$
\widetilde{\Phi}(x)=\Phi_{0} \frac{\sin \left(\frac{\omega x}{c}\right)}{\sin \left(\frac{\omega L}{c}\right)}
$$




\section{Resonancia en una cuerda real}

De acuerdo con la ecuación (14), la amplitud de respuesta se hace infinitamente grande si la frecuencia de la fuerza impulsora $\omega$ coincide con una de las frecuencias naturales de oscilación de la cuerda, esto es, cuando

$$
\omega=\omega_{n}=n \pi \frac{c}{L}
$$

Sin embargo, la existencia de fuerzas disipativas eliminan estos infinitos como veremos en la próxima sección. En una cuerda no ideal cuando la frecuencia de oscilación del extremo forzado está próxima a una de las frecuencias naturales del sistema, la amplitud de oscilación es muy grande y la cuerda adopta la forma del modo correspondiente. A este fenómeno se lo denomina resonancia.

\subsection{Resonancia en una cuerda con pérdidas}

En presencia de disipación la respuesta resonante tiene lugar para las frecuencias $\omega$ que minimizan el módulo del denominador de la ecuación (13).

Comosinh $(\gamma L)=\sinh (\alpha L) \cos (k L)+i \cosh (\alpha L) \sin (k L)$, se tiene que

$$
\begin{gathered}
\frac{1}{|\sinh (\gamma L)|}= \\
\frac{1}{\sqrt{\left[\sinh (\gamma L) \cos \left(\frac{\omega L}{c}\right)\right]^{2}+\left[\cosh (\alpha L) \sin \left(\frac{\omega L}{c}\right)\right]^{2}}}
\end{gathered}
$$

En la Fig. 4 se muestran gráficas de

$$
\left|\widetilde{\Phi}\left(x^{*}\right)\right|=\Phi_{0}\left|\frac{\sinh \left(\gamma x^{+}\right)}{\sinh (\gamma L)}\right|
$$

para dos valores diferentes de la constante de atenuación $\alpha$ en un punto $x^{*}$ cualquiera de la cuerda. Estas gráficas son las denominadas curvas de respuesta de transferencia (en el sentido que se registra el movimiento de una parte del sistema, $x=x^{*}$ que no coincide con el punto de excitación, $x=L$ ). Los picos en estas curvas son los picos de resonancia, o simplemente las resonancias del sistema. Las frecuencias en las que aparecen estos picos son las frecuencias de resonancia. Observemos que las frecuencias de resonancias son poco sensibles respecto de las variaciones del coeficiente de atenuación y se hallan, por lo tanto, muy próximas de las frecuencias resonantes del caso sin pérdidas. Sin embargo, la amplitud de la resonancia y su ancho de banda en frecuencia dependen fuertemente de la constante de atenuación.

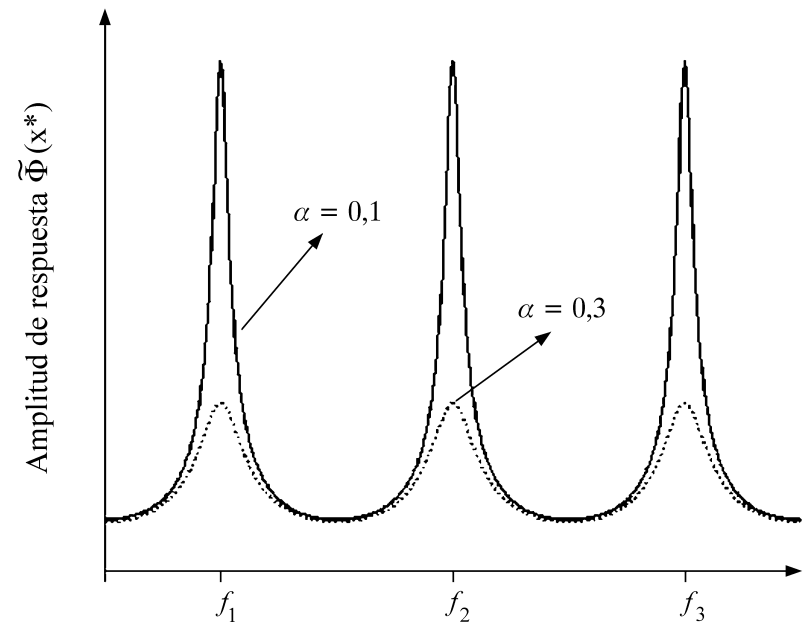

Frecuencia $(\mathrm{Hz})$

Figura 4: Gráficas de la amplitud de respuesta, $\left|\widetilde{\Phi}\left(x^{+}\right)\right|$, en función de la frecuencia para dos valores diferentes del coeficiente de atenuación.

Si la disipación es pequeña, $(\alpha L<<1, \sinh (\alpha L) \simeq \alpha L \mathrm{y}$ $\cosh (\alpha L) \simeq 1)$, la ecuación (16) se reduce a

$$
\frac{1}{|\sinh (\gamma L)|}=\frac{1}{\sqrt{(\alpha L)^{2} \cos ^{2}\left(\frac{\omega L}{c}\right)+\sin ^{2}\left(\frac{\omega L}{c}\right)}}
$$

En un pequeño entorno alrededor de la frecuencia natural de oscilación $\omega_{n}$ (ver ecuación 15), $|\cos (\omega L / c)| \simeq 1$ $\mathrm{y}|\sin (\omega L / c)| \simeq\left|\omega-\omega_{n}\right| L / c$, se tiene que

$$
\frac{1}{|\sinh (\gamma L)|}=\frac{c / L}{\sqrt{\frac{\Gamma^{2}}{4}+\left(\omega-\omega_{n}\right)^{2}}}
$$

La ecuación (18) muestra que la amplitud de respuesta, en el entorno de la frecuencia de resonancia, es una lorentziana.

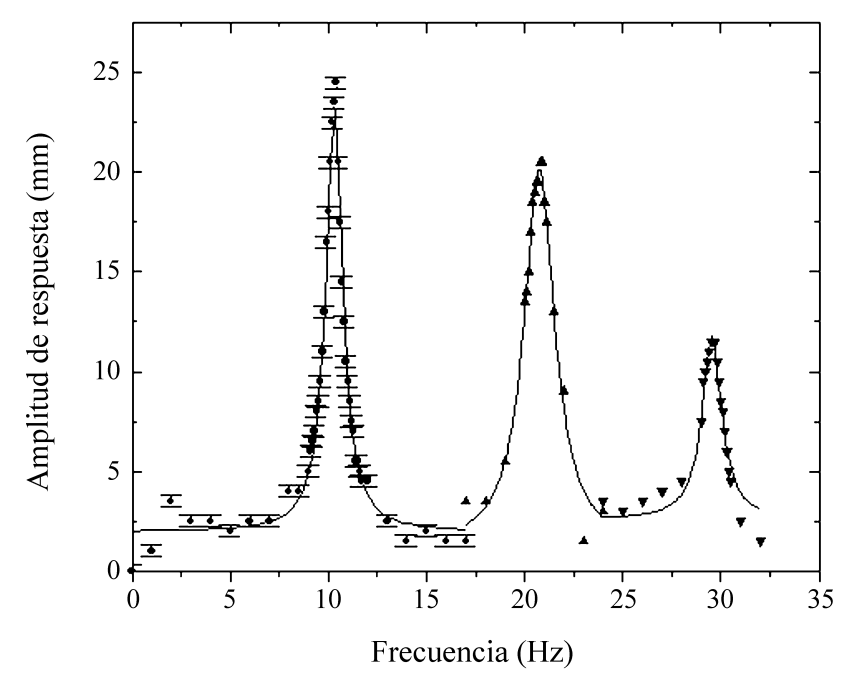

Figura 5: Gráfica experimental de la máxima amplitud de respuesta en función de la frecuencia. 
De la ecuación (18) se deduce igualmente que el ancho de banda en frecuencia (el intervalo de frecuencia en el cual la amplitud de respuesta es mayor que veces la amplitud máxima) de la resonancia es

$$
\Delta \omega=\Gamma
$$

En la Fig. 5 se muestran, los valores medidos, de la máxima amplitud de oscilación de la cuerda en función de la frecuencia. En el entorno de cada una de las frecuencias de resonancias, la curva que mejor ajusta los valores experimentales es una lorentziana (las curvas continuas en la Fig. 5). La diferencia de amplitud en los picos de resonancia se debe, en parte, a que la disipación depende de la frecuencia y, en parte, a que el vibrador no mantiene su amplitud $\Phi_{0}$ constante cuando se varía la frecuencia.

En la Fig. 5 se observa también que el ancho de banda en frecuencia es levemente diferente en las tres resonancias. Esto se explica solamente si $\Gamma$ (o sea $b$ ) depende de la frecuencia. En una situación real no se conoce a priori el valor de $b$, puesto que este coeficiente no está determinado solamente por las fuerzas viscosas con el aire (la ley de Stokes) sino, sobre todo, por las fuerzas de fricción internas para las cuales no existen expresiones analíticas. En la práctica el coeficiente $b$ se determina mediante la ecuación (19) y la curva de amplitud de respuesta del sistema como la que se muestra en la Fig. 5.

\section{Variación de la amplitud en función de la posición}

En la Fig. 6 se grafican los valores máximos (tanto positivos como negativos) de la amplitud de oscilación de los elementos de una cuerda de longitud $L=1 \mathrm{~m}$ que oscila en su tercer modo con un coeficiente de atenuación $\alpha=0,3$. Observemos que, en los nodos, la amplitud de la cuerda no es nula y que la amplitud de oscilación de los cuasi-nodos es más pequeña cuando más lejos está del punto de excitación. Se eligió un coeficiente de atenuación relativamente grande para que este hecho sea fácilmente observable en la Fig. 6.

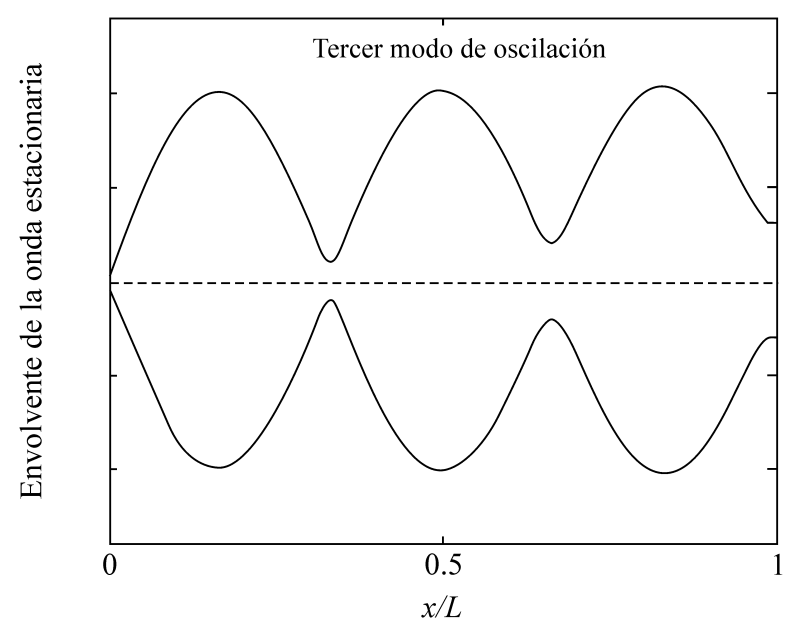

Figura 6: Envolvente de las oscilaciones de la cuerda oscilando en su tercer modo normal para $\alpha=0$ y $L=0$.

\section{Flujo de energía a lo largo de la cuerda}

La potencia que fluye a lo largo de cualquier punto $x$ de la cuerda en un cierto instante $t[3]$ viene dado por

$$
P(x, t)=-T \frac{\partial \Phi}{\partial x} \frac{\partial \Phi}{\partial t}
$$

Como estamos trabajando con fasores el valor medio (el promedio en el tiempo) de la potencia que fluye a lo largo del eje $x$ es

$$
\langle P(x, t)\rangle=-\frac{1}{2} \operatorname{Re}\left(-T \frac{\partial \Phi^{*}}{\partial x} \frac{\partial \Phi}{\partial t}\right)
$$

Reemplazando (13) en (20) y después de algunas operaciones algebraicas se obtiene

$$
\langle P(x, t)\rangle=-\frac{\omega}{2} \frac{T\left|\Phi_{0}\right|^{2}}{\sinh ^{2}(\gamma L)}[\beta \sinh (2 \alpha x)-\alpha \sin (2 \beta x)]
$$

Observemos que $\langle P(x, t)\rangle$ es siempre negativo salvo en ausencia de atenuación $(\alpha=0)$, en cuyo caso se tiene que $\langle P(x, t)\rangle=0$ para todo $x$. El signo negativo significa que la energía viaja, a lo largo de la cuerda, de derecha a izquierda, desde el vibrador (la fuente de energía) hacia el extremo fijo. Esta es la energía que, por unidad de tiempo, el vibrador entrega a la cuerda para mantener las oscilaciones. La disipación de energía a lo largo de la cuerda produce la disminución del valor medio del flujo de la energía, desde su máximo valor en $x=L$, hasta el extremo fijo, en $x=0$, donde $\langle P(0, t)\rangle=0$. Esto explica por qué en una cuerda con pérdidas los nodos no tienen amplitud cero, como en una cuerda ideal sin atenuación, y también por qué la amplitud de oscilación de estos "nodos" va disminuyendo cuando nos movemos desde el vibrador hacia el extremo fijo.

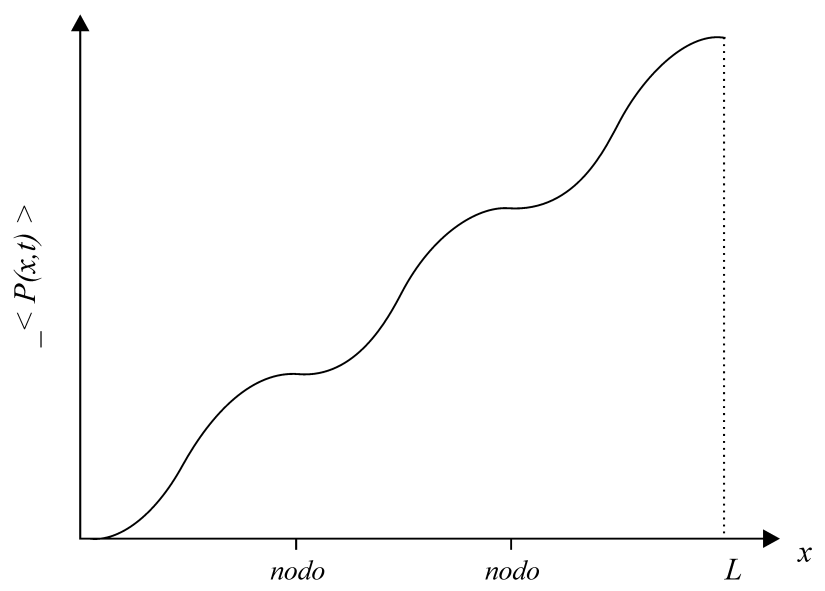

Figura 7: Gráfica de $-<P(x)>$ en función de $x$. La pendiente de esta curva es grande en los antinodos y muy pequeña en los cuasi-nodos. 
En la Fig. 7 se muestra una gráfica del módulo de la ecuación (21). Observemos que $|\langle P(x, t)\rangle|$ es monótona decreciente desde $x=L$ hasta $x=0$ y que la pendiente de esta curva es muy grande en un antinodo y muy pequeña en un cuasi-nodo. Es interesante interpretar físicamente las variaciones de la pendiente de esta curva.Para explicarlo utilizaremos la ecuación de conservación de la energía en un medio continuo unidimensional, [4][5],

$$
-\frac{\partial P}{\partial x}=\frac{\partial u}{\partial t}+p_{d}
$$

Esta ecuación expresa que la velocidad de decrecimiento de la potencia $P$ a lo largo de la cuerda es igual a la velocidad con que se incrementa la densidad de energía $u$ de la onda más la potencia disipada $p_{d}$ por unidad de longitud. Si tomamos el valor medio de la ecuación (22) obtenemos:

$$
\left\langle p_{d}\right\rangle=-\frac{\partial\langle P\rangle}{\partial x}
$$

(En el estado estacionario $u$ y su derivada con respecto al tiempo es una función periódica de $t$. Por lo tanto su promedio temporal es nulo).

La ecuación (23) nos dice que las variaciones de la pendiente de $-\partial\langle P\rangle / \partial x$ en la Fig. 7 se deben a que la disipación de energía es más grande en un antinodo que en un cuasi-nodo.

\section{Conclusión}

Tanto docentes como investigadores acuerdan en que los trabajos prácticos de laboratorio son muy útiles en la enseñanza aprendizaje de la Física [6][7] y que ésta debe enseñarse con actividades creativas y modelizaciones adecuadas para ayudar a salvar la brecha entre la Física enseñada y la Física real.

Consideramos que el trabajo práctico que describimos cumple esos requisitos. El acuerdo entre las mediciones, tanto de la amplitud de respuesta de las oscilaciones forzadas como de las variaciones de las amplitudes de los cuasi-nodos, con las predicciones del modelo simple que hemos desarrollado, son sorprendentes.

Esta experiencia permite, además, introducir el concepto de amplitud de respuesta, o curva de resonancia del sistema, que relaciona la entrada (fuerza impulsora) y la salida (amplitud de las oscilaciones de un dado punto de la cuerda) en función de la frecuencia. La amplitud de respuesta de la cuerda puede considerarse como una sucesión de curvas de resonancias de sistemas de un solo modo. La medición del ancho de banda en frecuencia de cada uno de los picos permite determinar el coeficiente de atenuación y su eventual dependencia de la frecuencia.

\section{Referencias}

[1] G. Stokes, Transactional Cambridge Philosophical Society 9, 8 (1851). Reimpreso en Mathematical and Physical Papers, editado por G. Stokes (Cambridge University Press, Cambridge, 1981) .

[2] A. French, Vibraciones y Ondas (Editorial Reverté, Barcelona, 1982).

[3] I. Main, Vibration and Waves in Physics (Cambridge University Press, Cambridge, 1984).
[4] B. Adler, L. Chu y R. Fano, Electromagnetic Energy Transmission and Radiation (J. Wiley \& Sons, New York, London, 1960).

[5] R. Welti, Introducción a la Física de las Ondas (UNR Editora, Rosario, Argentina, 1999).

[6] J. Salinas y L. Colombo, Revista de Enseñanza de Física 5, 10 (1992).

[7] V. Capuano, B. Follari, M.T. Perrota, G. Dima, E. Gutiérrez y A.M. De La Fuente, Memoria del Encuentro Nacional de Profesores de Física, Córdoba, Argentina, 2001. p. 129.

\section{Anexo: Oscilaciones libres de una cuerda con pérdidas}

En este trabajo analizamos las soluciones forzadas de la ecuación de ondas (3) para compararlas con los resultados de nuestra experiencia. El comportamiento de la cuerda en el régimen forzado, en particular el ancho de banda en frecuencia de sus resonancias está relacionado con el coeficiente de amortiguamiento de sus oscilaciones libres. Es por lo tanto conveniente deducir los cambios que introduce una pequeña fuerza viscosa a las oscilaciones libres de una cuerda sujeta de sus dos extremos.

Las oscilaciones libres de la cuerda son las soluciones de la ecuación (3) que satisfacen las condiciones de contorno homogéneas

$$
\widetilde{\Phi}(0)=0 \quad y \quad \widetilde{\Phi}(L)=0
$$

La solución de la ecuación diferencial (3) que satisface la primera de estas condiciones de borde es

$$
\widetilde{\Phi}(x)=2 B \sinh (\gamma x)
$$

donde $B$ es una constante arbitraria y

$$
\gamma=\frac{1}{c} \sqrt{i \Gamma \omega-\omega^{2}}
$$

La segunda condición de borde impone que

$$
2 B \sinh (\gamma L)=0
$$

Esta condición se satisface si y solamente si

$$
\gamma L=\frac{L}{c} \sqrt{i \Gamma \omega-\omega^{2}}=i n \pi \quad n=1,2,3, \ldots
$$

Elevando al cuadrado y reordenando los términos tenemos que

$$
\omega^{2}-i \Gamma \omega-c^{2} k_{n}^{2}=0
$$

donde

$$
k_{n}=\frac{n \pi}{L}
$$

Las soluciones de esta ecuación cuadrática son:

$$
\omega_{n}=i \frac{\Gamma}{2} \pm \frac{1}{2} \sqrt{-\Gamma^{2}+4 c^{2} k_{n}^{2}} \quad n=1,2,3, \ldots
$$


Si la fuerza viscosa es pequeña, $\Gamma \ll 2 c k_{n}$, se tiene que (tomando solamente las frecuencias reales positivas):

$$
\omega_{n}=\omega_{n}^{\prime}+i \Omega
$$

donde

$$
\begin{gathered}
\Omega=\frac{\Gamma}{2}, \\
\omega_{n}^{\prime}=\omega_{n 0}-\frac{1}{2}\left(\frac{\Omega}{\omega_{n 0}}\right) \Omega
\end{gathered}
$$

En (31), $\omega_{n 0}=c k_{n}=c n \pi / L$ es la frecuencia natural de oscilación en el caso sin pérdidas.
Las pequeñas pérdidas, modifican las frecuencias naturales de oscilación $\omega_{n 0}$, introduciendo una parte imaginaria pequeña $\left(\Omega \ll \omega_{n 0}\right)$ y una modificación en la parte real aún más pequeña.

En conclusión, la amplitud de las oscilaciones libres decae lentamente con el tiempo y su frecuencia natural de oscilación se modifica en una cantidad que es un orden de magnitud menor que el amortiguamiento. El tiempo $\tau$ en el cual la energía de las oscilacions libres deace a 1/e de su energía inicial, o sea, el tiempo de vida medio de las oscilaciones es $\tau=\Gamma^{-1}$.

Si $\Gamma>2 \omega_{n 0}$, las vibraciones de la cuerda son sobreamortiguadas, esto es después de sacarla de su posición de equilibrio la cuerda vuelve a su posición de equilibrio sin realizar ninguna oscilación. 